\title{
The influence of coating on the sensor wiring in medical guide wires
}

\author{
N. Stefanova, T. Opitz, T. Rossner, T. Meiss, R. Werthschützky \\ Technische Universität Darmstadt, Institute of Electromechanical Design, \\ Merckstr. 25, 64283 Darmstadt, Germany \\ E-Mail: n.stefanova@emk.tu-darmstadt.de
}

\begin{abstract}
The force measurement at the instrument tip during minimally invasive interventions can not only reduce the risk of complications, but also reduce the treatment time. This is especially valid for heart catheterization, where a long thin catheter and guide wire are inserted into the coronary vessels for localization and dilation of stenosis. In order to reduce the friction forces in the vascular system, the guide wire is usually coated with biocompatible materials. In this work the influence of the coating material pebax 3533 is being examined to the insulated copper wires which transmit the sensor signal from the distal to the proximal end of the guide wire. The results of this study show that the dissolved pebax has no significant influence to the polyurethane insulation of the copper wires and it can be used as a coating material.
\end{abstract}

Key words: medical guide wire, coating, copper wire, force sensor, pebax

\section{Introduction}

The haptic feedback of the forces acting on the guide wire tip is very important in minimally invasive surgery, and especially in the diagnosis and therapy of coronary arteries.

In order to provide the surgeon with a haptic force feedback on the guide wire tip, the Institute of Electromechanical Design (EMK) is developing a haptic assistive system (HapCath). Interaction forces between the guide wire tip and the blood vessels wall have to be measured by integrated force sensor into the guide wire tip and should be amplified via actuators coupled back on the guide wire, (Fig. 1). This force feedback provides an assistive system for the surgeon, which mediates a tactile impression of the interaction forces in the vascular system, simplifies navigation of the wire, reduces the risk of complications, and shortens treatment time [1].

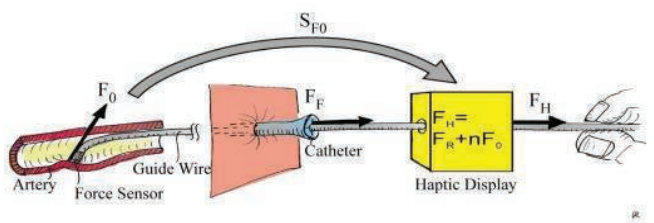

Fig. 1: Schematic of the haptic assistive system HapCath [1]

For measuring the force on the guide wire tip novel, miniaturized force sensors with piezo resistive principle are being developed [2]
Figure 2 shows the construction of a guide wire. The force sensor is integrated on the tip of the guide wire. Four insulated copper wires transfer the sensor signal to the connector. The wire core has diameter of $20 \mu \mathrm{m}$ and insulation of 5 $\mu \mathrm{m}$.

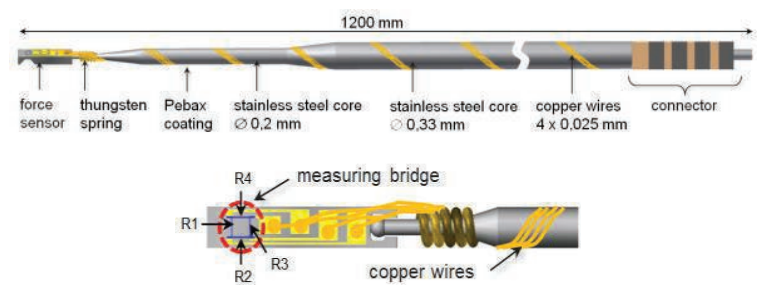

Fig. 2: Principle sketch of the guide wire

In order to keep the friction between the guide wire and blood vessel as low as possible, special coating is provided.

\section{Statement of the Problem}

The developed guide wire is to be coated with a suitable biocompatible material. Pebax 3533 is used as the coating material. It is a polyether block amide which is specifically designed for medical applications such as catheters, guide wires and tubes. The advantage of this material is low density, flexibility, excellent mechanical properties and good chemical resistance, [2]. The coating is applied on an in-line immersion system. The Pebax should be prepared accordingly for the selected coating process. The pebax is dissolved in a solvent with a certain viscosity. The solubility of pebax is very 
limited. The only solvent that can be used at room temperature is hexaflouroisopropanol (HFIP).

In order to protect the copper wires and the sensor from direct contact with the coating material, the guide wire is coated with a thin polyester shrink tubing.

The first experimental results show that the polyester shrink tubing is being damaged by the coating material, so that the wires are not further protected and are in fact in contact with the pebax. Chemically the raw pebax is not aggressive to plastics, however there is no information hereof for the solvent HFIP, with which it is liquefied. It is especially interesting to see how it affects polyurethane. In case of incompatibility with polyurethane, it may damage the wire insulation (Figure 2) and consequently come to electrical short. Since HFIP is used to solve its aggressiveness to plastics, especially to polyurethane, needs to be investigated.

Through this work, the interaction between the coating material pebax and the wire insulation is to be investigated. Afterwards its applicability should be evaluated for the mentioned guide wire.

\section{Implementation of Experiment}

In this study the following experiments were performed:

Experiment 1: The aim of this experiment is to see, if HFIP solvent damages the insulation of the wires. Here, the copper wires were first immersed for different period of time (5 minutes, 5 hours and 3 days) in the pure solvent (HFIP) (Figure 3, left). Then they were immersed in a solvent of nickel sulfate $\mu$ Chem 410 (SurTec Germany $\mathrm{GmbH}$ ). By applying a current of $2 \mathrm{~mA}$ nickel can be deposited in the electroplating bath at the places, where the insulation was damaged (Figure 3, right).

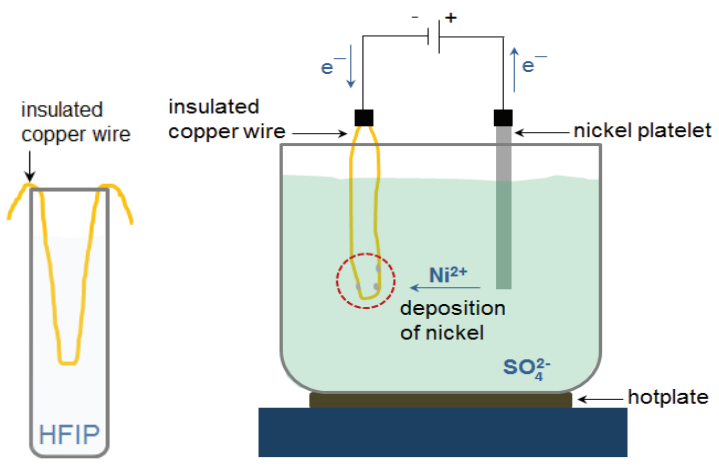

Fig. 3: Implementation of experiment, Left: insulated copper wire immersed in HFIP, Right: galvanizing of copper wire
Experiment 2: The aim of the second experiment is to investigate the influence of the dissolved Pebax on the wire insulation. The wires were wound around a stainless steel core and immersed three times in a pebax bath. Each immersion lasted 10 seconds and was followed by a one minute drying period. In order to test, if the insulation of the copper wires were damaged electrical short measurements were conducted, Figure 4.

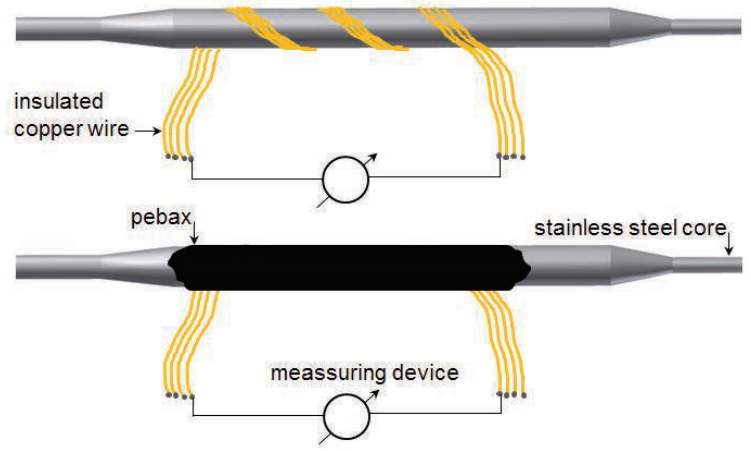

Fig. 4: Implementation of experiment, Above: copper wires coiled around a stainless steel core; Below: coated copper wires with pebax and check for short circuit

\section{Experimental Results}

The first experimental results show that the degree of damage of the wire insulation increases with the immersion time of the wires in the HFIP solvent. Figure 5 shows the microscope images of the galvanized copper wires. It can be clearly seen that the insulation of wire_1 (Figure 1a) was damaged more than wire_2 (Figure 1b). This can be recognized by the deposited nickel at the damaged places. In contrast the insulation of wire_2 (Figure 1c) was not damaged.

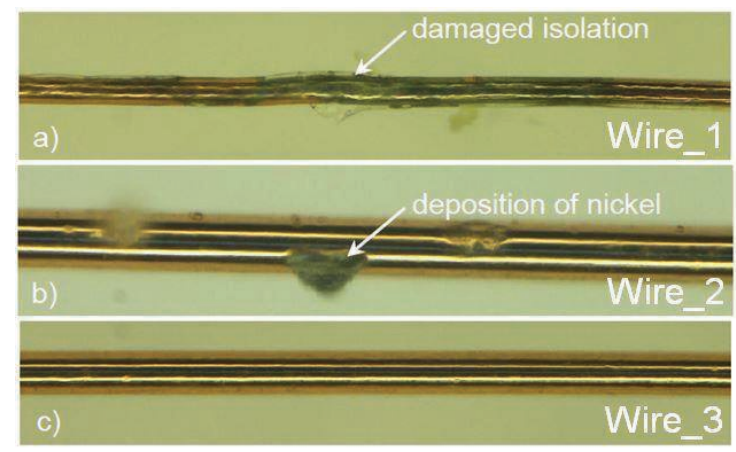

Fig. 5: Galvanized copper wires with different immersing time in HFIP, a) 3 days, b) 5 hours, c) 5 minutes

In order to evaluate the influence of pebax coating on the wiring, the current-voltage curve was recorded. In Figure 6 the current-voltage diagrams of the copper wires, shown in Figure 5 during the galvanizing are given. By the 
current-voltage diagrams the damage of the wire insulation revealed in Experiment 1 could be confirmed.
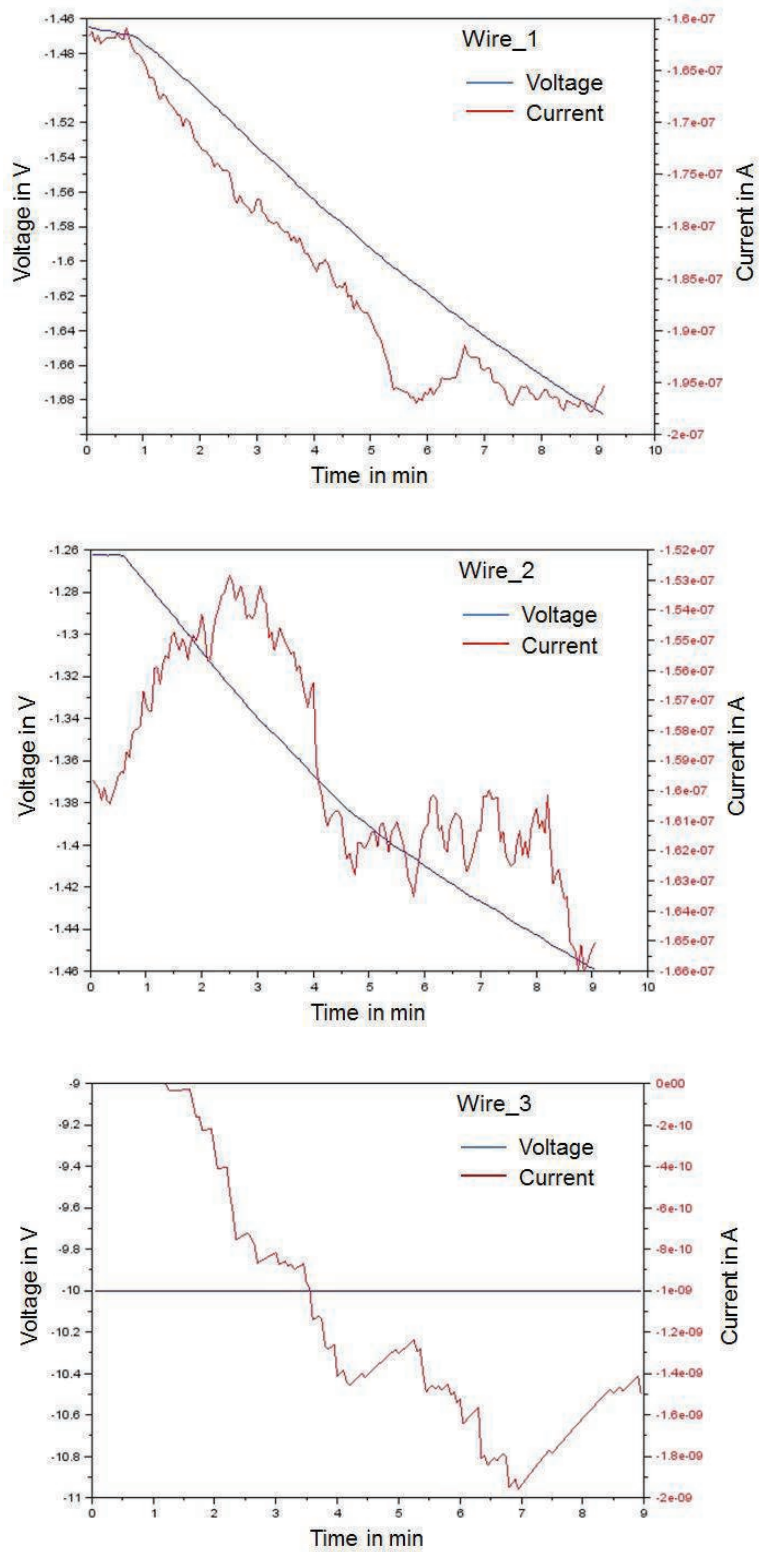

Fig. 6: Current-Voltage diagrams of copper wires during galvanization

As expected, the measured voltage in wire 1 was higher than in wire_2. The reason is that the wire insulation of both wires was damaged differently, due to the different duration of the immersion. The voltage increase over the time (see Figure 6, wire_1 and wire_2) can be explained by the deposited nickel layer on the damaged areas. Wire_3 voltage couldn't be measured because the wire insulation is intact.

The results of the second experiment show that the coating material is aggressive towards the polyester protective tube, but has no influence on the wire insulation. The reason is the high chemical resistance of the polyamide insulation, and the short interaction time due to the rapid evaporation of HFIP. Also, the coating viscosity does not influence the degree of damage of the wire insulation.

\section{Conclusions}

From the current-voltage diagram it can be seen that depending of the immersing time pure HFIP solvent caused damage to the sensor wiring. From the short time of immersion in the dissolved pebax the insulation of the copper wires was not damaged. In this study it could be proved that pebax 3533 can be used as a coating material for medical guide wires.

The next step is to investigate the influence of pebax on the force sensor by comparing the sensor measurement signal before and after coating with pebax.

\section{Acknowledgements}

We thank CIS Erfurt for help with the production of the force sensors and EPflex Feinwerktechnik for the support for the integration into practical guide wires. The project is funded under the number WE 2308 / 15-03 by the DFG.

\section{References}

[1] Meiss, T.: Konstruktion eines Mikrokraftsensors für Herzkatheterisierungen. Institut für Elektromechanische Konstruktion, Dissertation, TU Darmstadt, 2012.

[2] Meiss, T.; Werthschützky, R.: Konstruktion eines Mikrokraftsensors für Herzkatheterisierungen. Technisches Messen, Oldenburg Verlag, Vol. 76, Iss. 6, pp. 292-299.

[3] Elsner, P.; Eyerer, P.; Hirth, Th.: Kunststoffe: Eigenschaften und Anwendungen (Domininghaus). 8. neu bearbeitete und erweiterte Auflage, Springer Verlag, 2012, ISBN10: 3642161723. 